\title{
Diffraction in wide slits with semi-cylindrical edges
}

\author{
Philip Siegmann, Luis Miguel Sánchez-Brea, Juan Carlos Martínez-Antón, Eusebio Bernabeu \\ Dpto. Óptica, Facultad de Ciencias Físicas, Univ. Complutense de Madrid, 28040 Madrid, Spain
}

\begin{abstract}
We present an analytical model to obtain the diffraction pattern in far field of a metallic, thick slit based on the Geometrical Theory of Diffraction. The edges of the slit are modelled as semicylinders. We have considered that the thickness of the slit is sufficiently small compared to the width, so that the influence of multiple reflections between the edges may be neglected. The material in which the slit is made, as well as the polarization and angle of the incident beam, are considered. Notorious differences are obtained when compared to the classical diffraction from flat slits.
\end{abstract}

Key words: Diffraction - slit - geometrical theory of diffraction

\section{Introduction}

The slit is a well-known problem in diffraction theory. Commonly, slits are considered infinitely thin and with a perfect conductor behaviour, since the equations involved are much simpler [1] [2]. However, all real apertures present a certain thickness and a finite refraction index. In many situations, as in optical and microwave instrumentation, these approximations cannot be assumed, since thickness may modify the intensity distribution and the location of the diffraction minima and, therefore, make us to estimate wrongly the width of the slit.

Several works have been developed to determine the diffraction pattern of thick objects. For example, De Acetis [1] considers a perfectly conducting strip and he makes a comparison of Kirchhoff theory and the Geometrical Theory of Diffraction (GTD) [3] with the exact solution (rigorous solution of the Maxwell's equations) also by a large angle of incidence and width. They obtain that GTD are in better agreement with the exact solution than is Kirchhoff theory, especially in the region containing the secondary maxima. Other works that study diffraction by slits with rectangular edges or by semi-infinite plane are [4] [5] [6] [7]. The analytic solution obtained by Chu et al. for an cylinder-tipped half-plane using the GTD is in good

Received 3 September 2001; accepted 28 December 2001.

Correspondence to: P. Siegmann

Fax: ++34-913944674

E-mail: Siegmann@eucmos.sim.ucm.es agreement with the numerical results found from the exact solution in the spatial regions out from the geometrical transition region or shadow boundary (in these case Chu find an asymptotic solution that is applicable in these critical regions).

In this work, the diffraction pattern in far field for a slit with a certain thickness is determined by using the Geometrical Theory of Diffraction. It provides simple and accurate analytical results. This theory was originally proposed by Keller [8] as a generalization of geometrical optics: In addition to the usual geometrical rays reflected by the surface, other kind of rays, called surface rays, are considered for the determination of the diffraction pattern. These surface rays travel along the interface between two media. The diffraction pattern is then obtained as interference between surface and geometrical rays. In order to obtain the amplitudes and phases associated to the surface rays, a comparison of the GTD to the asymptotic development of the rigorous solution [3] [9] is carried out. This approximation allows us to incorporate, in an easy way, the effect of the reflection phenomena at the edges.

It is also necessary to model the shape of the edges, and we have considered them as semi-cylinders. We have assumed that the thickness of the slit $d$ is small compared to the aperture width $w$ (see fig. 1). In this case, the effect of multiple reflections between the

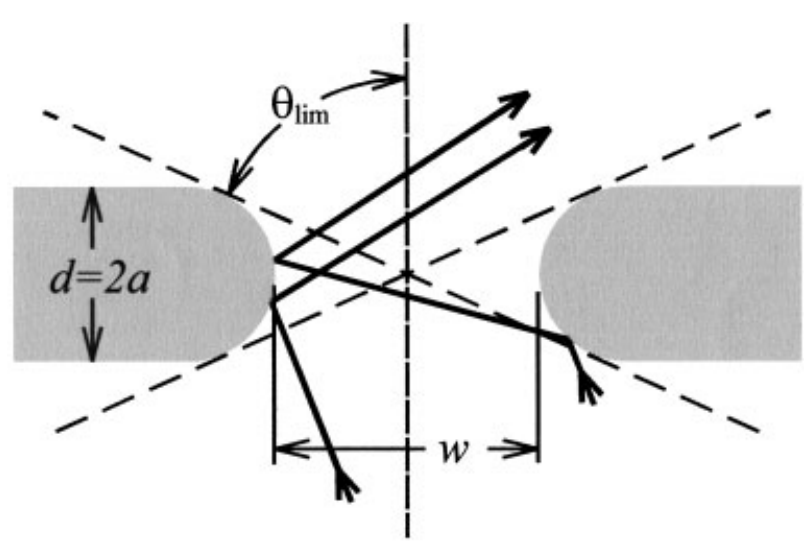

Fig. 1. Samples of rays which suffer one and two reflection. The rays, which suffer one reflection, are mostly contained in the fan represented here $\left(-\theta_{\lim } \geq \theta_{2} \geq \theta_{\lim }\right)$. 
edges is negligible and equations involved become even simpler. Multiple reflections may modify the diffraction pattern when this assumption is not fulfilled.

We have obtained that the diffraction pattern depends on the width and thickness of the slit, the material in which it is made, and also on the angle of the incident beam and its polarization. Furthermore, by a graphic comparison of our results with that obtained by the theory of Fraunhofer of a similarly flat slit, we appreciate a significantly difference.

\section{Model based on the Geometrical Theory of Diffraction}

Let us consider a metallic slit consisting of two semicylindrical edges with a radius $a$ and separated a distance $w$ (see fig. 2). The reflection index of the edges is $n_{c}$. This slit is illuminated with a monochromatic plane wave whose wavevector is containing in the $X Y$-plane, forming an incidence angle $\theta_{1}$ with the $y$-axis.

The field proposed then by the GTD is the sum of two fields: a geometrical field $U_{g}$ associated to the reflected rays, and a diffracted field $U_{\text {dif }}$ associated to the diffracted rays which travel along a geodesic on the convex edge surface into the shadow region. The incident field should also be considered but in the far field it only affects to $\theta_{2}=-\theta_{1}$, and it will be neglected. The values for the geometrical and diffracted fields are respectively [9]

$$
\begin{aligned}
U_{g}= & U_{0}(B) \cdot R^{s, p}(\gamma) \sqrt{\frac{a \cos \gamma}{2 s_{g}+a \cos \gamma}} \cdot \exp \left(\mathrm{i} k s_{g}\right) \\
U_{\mathrm{dif}}= & U_{0}(D) \cdot \sum_{m=1}\left(B_{m}^{s, p}\right)^{2} \frac{\exp \left(-\beta_{m}^{s, p} s_{\mathrm{dif}}^{\prime}\right)}{\sqrt{s_{\mathrm{dif}}}} \\
& \times \exp \left(\mathrm{i} k\left(s_{\mathrm{dif}}+s_{\mathrm{dif}}^{\prime}\right)\right)
\end{aligned}
$$

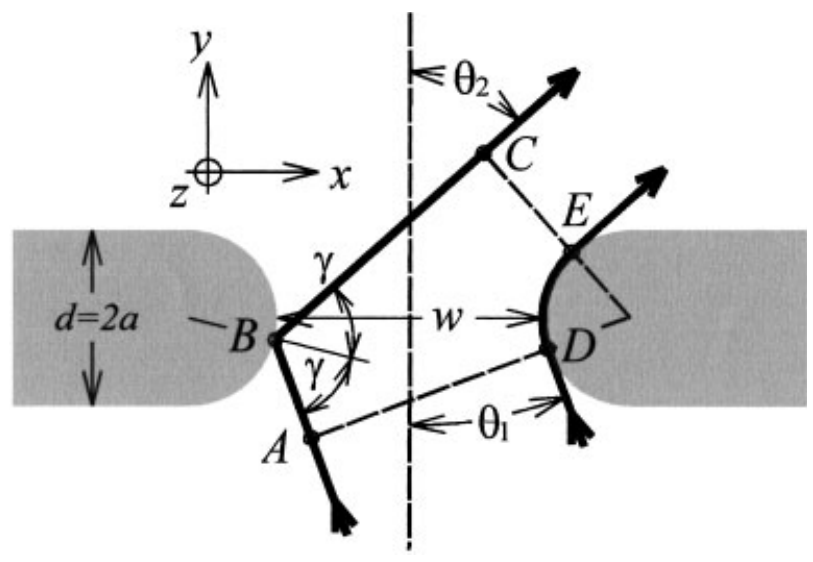

Fig. 2. Scheme of a thick slit with two semi-cylinders edges. The optical paths of the geometrical and diffracted rays, and the parameters involved are shown. where $s$ and $p$ stand for perpendicular and parallel polarization respect to the plane of incidence (parallel and perpendicular to the $z$ axis respectively), $B_{m}^{s, p}$ are the diffraction coefficients and $\beta_{m}^{s, p}$ the absorption coefficients, which are complex numbers tabulated in [9] (see appendix). $\gamma=\left(\pi-\left|\theta_{1}+\theta_{2}\right|\right) / 2$ is the local angle of incidence (see fig. 2). $R^{s, p}(\gamma)$ are the reflection coefficients given by Fresnel equations [10] which are dependent on the refractive indices of the metal $n_{c}$. $U_{0}$ is the incident field of the plane wave at the incident points $B$ and $D$, and $k$ is the wavevector. $s_{g}$ is the optical paths of the reflected geometrical rays, $s_{\text {dif }}^{\prime}$ is the length of the geodesic travelled by the surface rays before dear tangentially emission by $E$, and $s_{d i f}$ is the optical paths of the diffracted rays which are tangentially emitted by $E$.

Defining $s_{\mathrm{dif}} \equiv r$, and being $s_{g}=[B C]+r$, we can reduce de eq. (1) by considering $r \gg a$ (far field), to

$$
\begin{aligned}
U_{g} \rightarrow & \frac{U_{0} \exp (\mathrm{i} k r)}{\sqrt{r}} \cdot R^{s, p}(\gamma) \sqrt{\frac{a}{2} \cos \gamma} \\
& \times \exp (\mathrm{i} k([B C]+[A B]), \\
U_{\text {dif }} \rightarrow & \frac{U_{0} \exp (\mathrm{i} k r)}{\sqrt{r}} \cdot \sum_{m=1}\left(B_{m}^{s, p}\right)^{2} \exp \left(-\beta_{m}^{s, p} s_{\text {dif }}^{\prime}\right) \\
& \times \exp \left(\mathrm{i} k s_{\text {dif }}^{\prime}\right),
\end{aligned}
$$

where $U_{0}(B)=U_{0}(A) e^{i k[A B]}$ and $U_{0} \equiv U_{0}(A)=U_{0}(D)$. We see that far enough from the slit the reflected and diffracted rays go like cylindrical waves. The optical paths for the rays are

$$
\begin{gathered}
{[A B]+[B C]=(2 a+w)\left(\sin \theta_{1}+\sin \theta_{2}\right)} \\
-2 a \sin \left(\frac{\theta_{1}+\theta_{2}}{2}\right), \\
s_{\text {dif }}^{\prime}=a\left(\theta_{1}+\theta_{2}\right) .
\end{gathered}
$$

The summatory (over $m$ ) in the diffracted field has been interpreted as "layers" or rays which travel at a certain distance $\delta_{m}^{s, p}$ to the surface, called surface rays (see appendix). The concept of layer comes from Hönl [9] when comparing the asymptotic solution with that obtained by the GTD. The diffraction pattern can be easily determined as the interference between these two fields from eq. (2); $I=\left|U_{\mathrm{dif}}+U_{g}\right|^{2}$ resulting with eq. (3)

$$
\begin{aligned}
& I\left(\theta_{2}\right) \\
& =\frac{I_{0}}{r}\left\{\begin{array}{l}
\left|R^{s, p}\right|^{2} \frac{a}{2} \cos \gamma+\left|R^{s, p}\right| \sqrt{\frac{a}{2} \cos \gamma} \\
\times \sum_{m} \Omega_{m}^{s, p} \cos \left(k \Delta-v_{m}^{s, p}+\varrho^{s, p}\right)+\sum_{m}\left(\Omega_{m}^{s, p}\right)^{2} \\
+2 \sum_{m} \sum_{n=m+1} \Omega_{m}^{s, p} \Omega_{n}^{s, p} \exp \left(v_{m}^{s, p}-v_{n}^{s, p}\right)
\end{array}\right\},
\end{aligned}
$$




$$
\begin{aligned}
& \text { where } I_{0}=\left(U_{0}\right)^{2} \text { and } \\
& \begin{aligned}
\Delta=|[A C]-[D E]|, \\
R^{s, p}=\left|R^{s, p}\right| \exp \left(\mathrm{i} \varrho^{s, p}\right), \\
\Omega_{m}^{s, p}=\left|\left(B_{m}^{s, p}\right)^{2}\right| \exp \left(-\operatorname{Re}\left[\beta_{m}^{s, p}\right] s_{\text {dif }}^{\prime}\right), \\
v_{m}^{s, p}=\phi-\operatorname{Im}\left[\beta_{m}^{s, p}\right] s_{\text {dif }}^{\prime}, \quad \phi=\arg \left[\left(B_{m}^{s, p}\right)^{2}\right],
\end{aligned}
\end{aligned}
$$

being $\phi$ independent on $m, s$ and $p$.

Higher terms in the summatory of eq. (4) affect mainly to angles close to the incidence angle $\theta_{2}=-\theta_{1}$ corresponding to the Absolute Diffraction Maximum (ADM). Therefore, if we are not interested in the nearby of the ADM we can use few terms for an accurate determination of the diffraction pattern. The effect of the higher terms is even smaller when the radius of the semi-cylinders is much larger than the wavelength. This way, if $a \gg \lambda$, or are we sufficiently away from the ADM eq. (4) can be written as

$$
I\left(\theta_{2}\right) \cong \frac{I_{0}}{r}\left\{\begin{array}{l}
\left|R^{s, p}\right|^{2} \frac{a}{2} \cos \gamma+\left|\left(B_{1}^{s, p}\right)^{2}\right|^{2} \\
\times \exp \left(-2 \operatorname{Re}\left[\beta_{1}^{s, p}\right] s_{\text {dif }}^{\prime}\right)+\left|R^{s, p}\right|\left|\left(B_{1}^{s, p}\right)^{2}\right| \\
\times \exp \left(-2 \operatorname{Re}\left[\beta_{1}^{s, p}\right] s_{\text {dif }}^{\prime}\right) \sqrt{\frac{a}{2} \cos \gamma} \\
\times \cos \left(k \Delta-v_{1}^{s, p}+\varrho^{s, p}\right)
\end{array}\right\}
$$

where we have taken only the first term of the summatory.

We consider then, that the formulation presented in the eq. (4) is worth for slits with cylindrical edges whose thickness is sufficiently thin in comparison with the aperture width. Nevertheless for the case of a strip and when higher order terms are included, the GTD yields a good prediction of the diffraction pattern even for strip widths down to one wavelength or less [1]. The simplified eq. (5) also considers dispersion angles far from the ADM.

Furthermore we despise the effect of multi-reflected rays taking $d \ll w$. The range for the observation angle $\Delta \theta_{2} \in\left(-\theta_{\lim }, \theta_{\lim }\right)$ in which the once-reflected rays are contained, is approximately the fan shown in fig. 1. This angle $\Delta \theta_{\text {lim }}$ is

$$
\theta_{\lim }=\frac{\pi}{2}-\arcsin \left(\frac{2 a}{2 a+w}\right) .
$$

In the following theoretical results we will then restrict ourselves to this limit of validity of the pattern for $\theta_{2}$ between $-\theta_{\text {lim }}$ and $\theta_{\text {lim }}$.

We want to compare our results with the Fraunhofer diffraction pattern for a plane slit, which has the following formula [10]

$$
I_{F h}\left(\theta_{2}\right)=I_{0} K^{2} \frac{\sin ^{2}\left(\frac{k a}{2}\left(\sin \theta_{2}+\sin \theta_{1}\right)\right)}{\left(\frac{k a}{2}\left(\sin \theta_{2}+\sin \theta_{1}\right)\right)^{2}},
$$

where we have incorporated the obliquity factor $K=\left(\cos \theta_{2}+\cos \theta_{1}\right) / 2$.

\section{Results}

In order to determine the behaviour of the diffraction pattern of a slit with a certain thickness, we have simulated several cases and we have analysed how the diffraction pattern is modified with respect to the „Fraunhofer slit". Due to the ignorance of the strict value of the intensity in the ADM (there will be necessary to consider infinite layers) we couldn't normalize the diffraction pattern to compare it with Fraunhofer, so we have allowed ourselves to multiplicity in eq. (7) by a appropriate $I_{0}$ which facilitate the comparison of the two pattern.

The number of layers or terms considered in the eq. (4) to obtain the following results are the minima necessary to produce a significant change in the diffraction pattern in a certain angular interval. In any case, the maximum number of layers calculated was 122.

Firstly, in fig. 3, we represent the diffraction pattern of a slit with a certain thickness (obtained with eq. (4)), in comparison to the Fraunhofer plane slit with the same width $w$. The GTD diffraction pattern of a thick slit turns out to be more realistic than that given by Fraunhofer. By the GTD the contribution of the geometric field produces a progressive increase of the strong fluctuation of the intensity with $\theta_{2}$ while be the Fraunhofer pattern the intensity fluctuation goes to zero with increasing $\theta_{2}$.

In fig. 4 , the influence of the number of terms considered in the summatory of eq. (4) is shown. Close to the ADM, the term that contributes more is the diffracted part $U_{\text {dif }}$. The more far away are these layers which form the surface rays from the surface of the

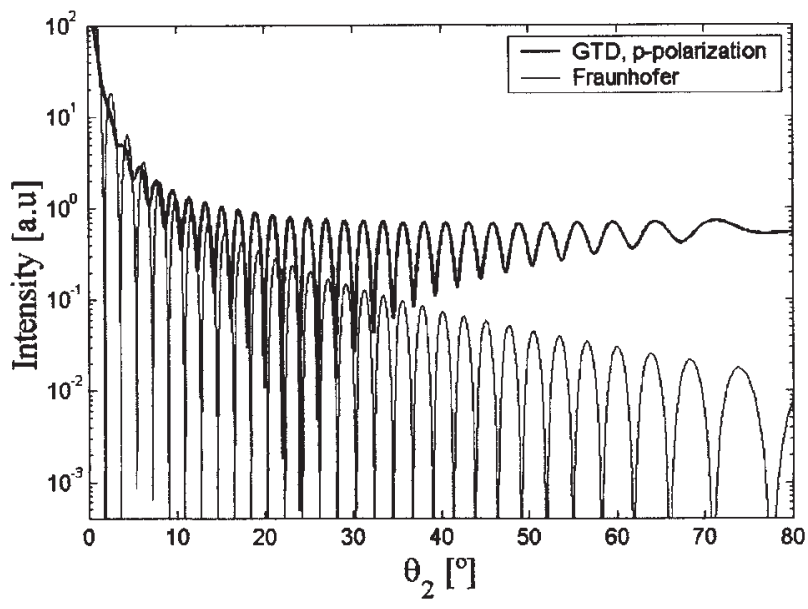

Fig. 3. Diffraction pattern in logarithmic scale from a perfectly conducting slit with $w=20 \mu \mathrm{m}, a=2 \mu \mathrm{m}$. The incident beam was $p$ polarized with $\lambda=0.630 \mathrm{~mm}$ and incident angle $\theta_{1}=0^{\circ}$. We have compared two models: GTD without consideration of multiple reflection (thick line), and Fraunhofer diffraction from a plane slit with aperture $w$ (thin line) and $I_{0}=0.04$ a.u. We have considered even 122 terms in the summatory of the equation eq. (4). 


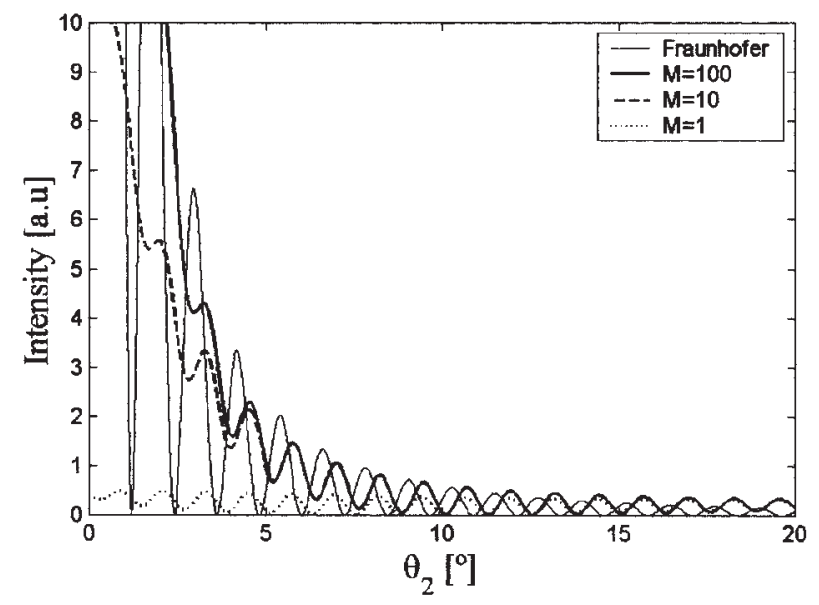

Fig. 4. Influences of the number of layers or superficial rays $(M=1,10$ and 100) in the diffraction pattern of a gold slit $\left(n_{c}=2.82+\mathrm{i} \cdot 0.37\right)$ with to $a=3 \mu \mathrm{m}$ and $w=30 \mu \mathrm{m}$. The wavelength of the incident plane wave was also $\lambda=0.630 \mu \mathrm{m}$, with polarization $s$ and angle of incidence $0^{\circ}$. Comparison with Fraunhofer diffraction for a plane slit with the same width $w$ $\left(I_{0}=0.018\right.$ a. u. $)$.

edges, so much less energy goes to the diffracted rays and so more contribute these surface rays to the $\mathrm{ADM}$, affecting even less to the rest of the diffraction pattern. This effect is softer if the radius of curvature seems to the wavelength. In these area close to the $\mathrm{ADM}$ we can see, if we considerate even more terms, that the fall of the envelope of secondary maxima of the GTD becomes similar to the fall of the evelope of the secondary maxima obtained by the plane slit from Fraunhofer.

The region close to the ADM, where the GTD fails, is known as the shadow boundary transition region, and there the ray optical field description changes rapidly, but continuously, from the geometrical optic field to the surface diffracted field (this discontinuously can be overcame by the Uniform Theory of Diffraction (UTD) [3]). But experimentally is not easy to determine the intensity distribution close to the diffraction maxima, and in the rest of angles, both approaches are similar.

In fig. 5 we show the diffraction pattern, for $s$ and $p$ polarization when the incidence angle is $\theta_{1}=-20^{\circ}$. The slit is made of aluminium and $a=3 \mu \mathrm{m}$, and $w=30 \mu \mathrm{m}$. We have considered 122 layers in order to estimate properly the vicinity of the ADM. We see that there is an important difference with the Fraunhofer pattern, not only in the amplitude, but also in the location of the minima. Here we also have multiply with an appropriate $I_{0}$ in the eq. (7) to facility the comparison.

Fig. 6 shows the effect of the incident beam polarization and the material (aluminium and iron). The polarization is an important factor that modifies the diffraction pattern. Not only the location of the minima changes with polarization, but also the intensity distribution of the diffraction pattern. The material changes

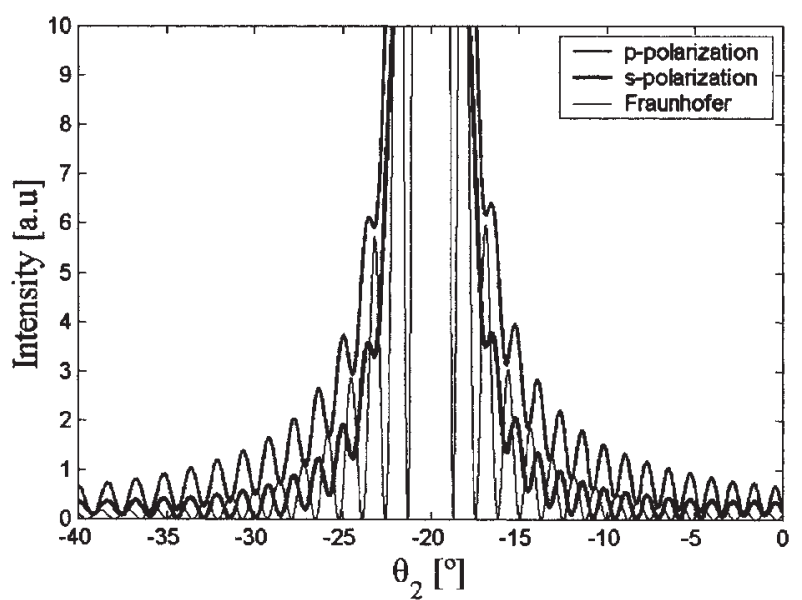

Fig. 5. Diffraction pattern close to the ADM of a aluminium slit $\left(n_{c}=1.63+\mathrm{i} \cdot 1.51\right)$ with $w=30 \mu \mathrm{m}$ and $a=3 \mu \mathrm{m}$. The incident beam have an incidence angle $\theta_{1}=-20^{\circ}$ and $\lambda=0.630 \mu \mathrm{m}$. We distinguish polarizations $s$ and $p$ for the fig. obtained by the GTD and compare it with the one obtained by Fraunhofer for a plane slit with the same width and where $I_{0}=0.018$ a.u. The number of layers considered by the GTD was 122 .

the reflectivity and the phase, and as a consequence, also the location of the diffraction minima, what is important to know if we want to determine geometrical information about the slit from their diffraction pattern.

In fig. 7, we have simulated several slits with equal width, but with a different thickness. In this case not only the envelopes of the diffraction pattern change, but also the location of the minima. This effect indicates us that, when measuring the width of the slit

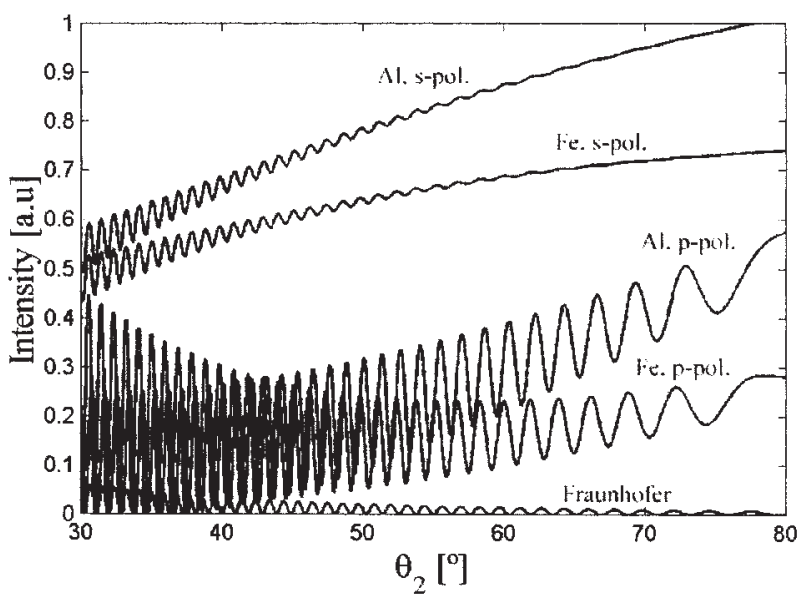

Fig. 6. Diffraction patterns for two slits of different materials: iron $\left(n_{c}=1.63+\mathrm{i} \cdot 1.44\right)$ and aluminium $\left(n_{c}=5.23+\mathrm{i} \cdot 1.44\right)$, width wide $w=50 \mu \mathrm{m}$ and thickness $2 a=10 \mu \mathrm{m}$. The slit was illuminated with an monochromatic beam $(\lambda=0.630 \mu \mathrm{m})$ in normal incidence for $s$ - and $p$-polarization. We consider only 1 layer. We also represent the Fraunhofer diffraction pattern where $I_{0}$ was taken equal to 0.018 a.u. 


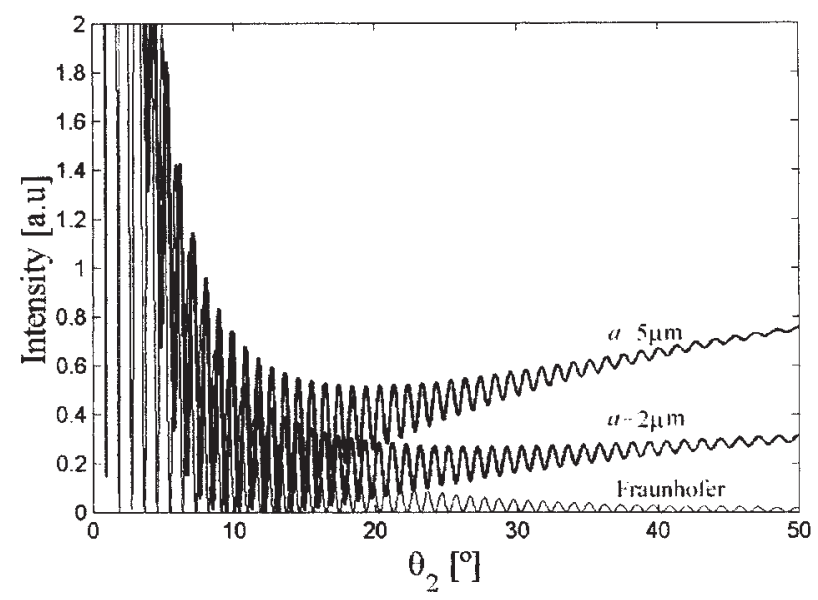

Fig. 7. Diffraction patterns of two slits $(2 a=4$ and $10 \mu \mathrm{m})$. The material of the slits are steel $\left(n_{c}=3.37+\mathrm{i} \cdot 2.27\right)$, the aperture $w=40 \mathrm{~mm}$ and the polarization of the incident beam $s$, with $\lambda=0.630 \mu \mathrm{m}$ and $\theta_{1}=0^{\circ}$. The number of layers considered in eq. (4) was 10 . In (thick line) are represented the Fraunhofer diffraction pattern where $I_{0}$ was taken equal to 0.015 a.u.

form the diffraction minima, the thickness of the slit needs to be considered, because it modifies the location of the minima and, therefore, the width estimation. It can also by seen that the thick slit contributes with a greater geometrical field $U_{g}$, i.e., the contribution to the field of the reflected rays is greater for thick slits, while for a plane slit (Fraunhofer) this contribution doesn't exist.

We have also analysed how the diffraction pattern changes with the width of the slit $w$, as it is shown in fig. 8. It is well known that for thin slits the distance between the diffraction minima decreases inversely to the width. This behaviour is also present when the slit

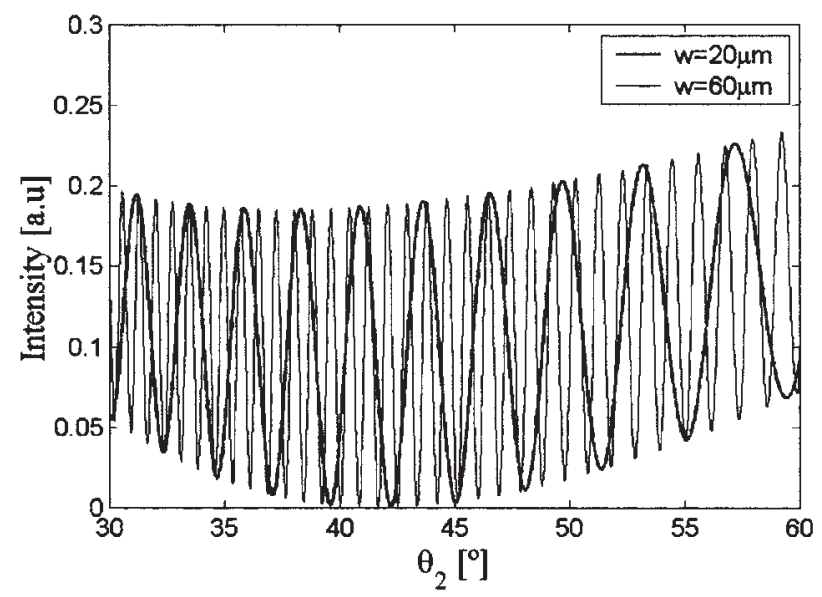

Fig. 8. Comparison between the diffraction patterns of two slits with different widths: $w=20 \mu \mathrm{m}$ and $60 \mu \mathrm{m}$. The other slit parameters are: $n_{c}=4.66+i \cdot 1.93$ (zinc) and $2 a=6 \mu \mathrm{m}$. The incident beam is $p$-polarized, with $\lambda=0.630 \mu \mathrm{m}$ and $\theta_{1}=0^{\circ}$. The number of layers in eq. (4) was 10. is thick, as it can be shown in fig. 7. In this figure the diffraction pattern of two slits with widths $w=30$ and $60 \mu \mathrm{m}$, made of steel and with a thickness $2 a=6 \mu \mathrm{m}$ are shown. It is observed that the distance between minima is different for the two slits, but the envelopes, and therefore the contrast, are the same. To obtain this figure we used eq. (4) with 10 layers.

\section{Conclusion}

In this work we have analysed, by means of the Geometric Theory of the Diffraction, the intensity distribution generated by a slit with a certain thickness, which has been modelled using semi-cylindrical edges. According to this model, the diffracted intensity depends, not only on the width of the slit, but also on its depth, the polarization of the beam and on the material in which it is made.

\section{Appendix}

The exponential terms in the summatory by the second equation from eq. (2) can be written in terms of the distance of the surface rays to the surface [9] (resulting by comparison of the GTD to the asymptotic development of the rigorous solution):

$$
\begin{aligned}
& \exp \left(\mathrm{i} k s_{\mathrm{dif}}^{\prime}\right) \cdot \exp \left(-\beta_{m}^{s, p}\right) \\
& =\exp \left[\mathrm{i} k\left(a+\delta_{m}^{s, p}\right)\left(\theta_{1}+\theta_{2}\right)\right] \cdot \exp \left[-k \delta_{m}^{s, p} \sqrt{3}\left(\theta_{1}+\theta_{2}\right)\right] .
\end{aligned}
$$

Herewith we see that to the radius $a$ is added an additional term $\delta_{m}^{s, p}$ which can be visualised as a distance of the $m^{\text {th }}$ surface ray to the surface. The distances $\delta_{m}^{s, p}$ are related with the absorption coefficient $\beta_{m}^{s, p}$ by:

$$
\delta_{m}^{s, p}=\frac{a}{k} \frac{\beta_{m}^{s, p}}{\sqrt{3}-\mathrm{i}} .
$$

The values of the absorption coefficients and of the diffraction coefficients $\left(B_{m}^{s, p}\right)$ are also obtain from the rigorous solution and are

$$
\beta_{m}^{s, p}=\frac{\sqrt{3}-\mathrm{i}}{2 a}\left(\frac{k a}{6}\right)^{1 / 3} q_{m}^{s, p}, \quad B_{m}^{s, p}=\frac{a^{1 / 3} C_{m}^{s, p}}{\sqrt{2} k^{1 / 6}} e^{\frac{\mathrm{i} \pi}{12}},
$$

where $m$ indicate the layer, $q_{m}^{p}$ are the zeros of the Airy Integral $(A(q))$ and $q_{m}^{s}$ the zeros of the derivation of the Airy Integral $\left(A^{\prime}(q)\right)$. The terms $C_{m}^{s, p}$ are the amplitude of the surface rays given by

$$
C_{m}^{s}=\frac{\pi^{3 / 2}}{3 \cdot 6^{1 / 3}\left(A^{\prime}\left(q_{m}^{s}\right)\right)^{2}}, \quad C_{m}^{p}=\frac{\pi^{3 / 2}}{6^{1 / 6}\left(A\left(q_{m}^{p}\right)\right)^{2}} .
$$

In table 1 we give some value of the coefficients for each polarization and for a perfect convex conductor with radio of curvature $a=100 \mu \mathrm{m}$ and for an incident wave with $\lambda=0.630 \mu \mathrm{m}$. 
Table 1. GTD coefficients for $s$ and $p$ polarization, for a perfect convex conductor with radius of curvature $a=100 \mu \mathrm{m}$ and $\lambda=0.630 \mu \mathrm{m}$.

\begin{tabular}{|l|l|l|l|l|l|l|l|l|l|l|}
\hline$m$ & $q_{m}^{s}$ & $q_{m}^{p}$ & $C_{m}^{s}$ & $C_{m}^{p}$ & $B_{m}^{s}\left[\mu \mathrm{m}^{1 / 2}\right]$ & $B_{m}^{p}\left[\mu \mathrm{m}^{1 / 2}\right]$ & $\beta_{m}^{s}\left[\mu \mathrm{m}^{-1}\right]$ & $\beta_{m}^{p}\left[\mu \mathrm{m}^{-1}\right]$ & $\delta_{m}^{s}[\mu \mathrm{m}]$ & $\delta_{m}^{p}[\mu \mathrm{m}]$ \\
\hline 1 & 3.3721 & 1.4694 & 0.9107 & 1.5318 & $\begin{array}{l}1.9679+ \\
\mathrm{i} \cdot 0.5273\end{array}$ & $\begin{array}{l}3.3100+ \\
\mathrm{i} \cdot 0.8869\end{array}$ & $\begin{array}{l}0.1606- \\
\mathrm{i} \cdot 0.0927\end{array}$ & $\begin{array}{l}0.0700- \\
\mathrm{i} \cdot 0.0404\end{array}$ & 0.9295 & 0.4050 \\
\hline 2 & 5.8958 & 4.6847 & 0.6943 & 0.7852 & $\begin{array}{l}1.5002+ \\
\mathrm{i} \cdot 0.4020\end{array}$ & $\begin{array}{l}1.6967+ \\
\mathrm{i} \cdot 0.4546\end{array}$ & $\begin{array}{l}0.2807- \\
\mathrm{i} \cdot 0.1621\end{array}$ & $\begin{array}{l}0.2231- \\
\mathrm{i} \cdot 0.1288\end{array}$ & 1.6252 & 1.2913 \\
\hline 3 & 7.9620 & 6.9518 & 0.5982 & 0.6420 & $\begin{array}{l}1.2926+ \\
\mathrm{i} \cdot 0.3464\end{array}$ & $\begin{array}{l}1.3872+ \\
\mathrm{i} \cdot 0.3717\end{array}$ & $\begin{array}{l}0.3791- \\
\mathrm{i} \cdot 0.2189\end{array}$ & $\begin{array}{l}0.3310- \\
\mathrm{i} \cdot 0.1911\end{array}$ & 2.1947 & 1.9163 \\
\hline 4 & 9.7881 & 8.8890 & 0.5397 & 0.5672 & $\begin{array}{l}1.1663+ \\
\mathrm{i} \cdot 0.3125\end{array}$ & $\begin{array}{l}1.2256+ \\
\mathrm{i} \cdot 0.3284\end{array}$ & $\begin{array}{l}0.4661- \\
\mathrm{i} \cdot 0.2691\end{array}$ & $\begin{array}{l}0.4233- \\
\mathrm{i} \cdot 0.2444\end{array}$ & 2.6981 & 2.4503 \\
\hline 5 & 11.4574 & 10.6325 & 0.4990 & 0.5184 & $\begin{array}{l}1.0782+ \\
\mathrm{i} \cdot 0.2889\end{array}$ & $\begin{array}{l}1.1202+ \\
\mathrm{i} \cdot 0.3002\end{array}$ & $\begin{array}{l}0.5456- \\
\mathrm{i} \cdot 0.3150\end{array}$ & $\begin{array}{l}0.5063- \\
\mathrm{i} \cdot 0.2923\end{array}$ & 3.1582 & 2.9303 \\
\hline$\vdots$ & $\vdots$ & $\vdots$ & $\vdots$ & $\vdots$ & $\vdots$ & $\vdots$ & $\vdots$ & $\vdots$ \\
\hline
\end{tabular}

\section{References}

[1] DeAcetis LA, Einstein FS, Juliano RA Jr., Lazar I: Single strip diffraction: comparison of Kirchhoff theory and geometrical theory with the exact solution in the limit of small glancing angle and width; perpendicular polarization. Appl. Opt. 15 (1976) 2866-2870

[2] Mayes TW, Melton BF: Fraunhofer diffraction of visible light by a narrow slit. Am J. Phys. 62 (1994) 397-403

[3] Skwirzynski JK: Proceedings of the NATO advanced study institute on theoretical methods for determining the interaction of electromagnetic waves with structures. Sijthoff \& Noordhoff, Alphen aan den Rijn, the Netherlands 1981

[4] Chu YT, Anderson VE, Hubbell HH Jr., Ferrell TL: Asymptotic solution for the diffraction of an electromagnetic plane wave by cylinder-tipped half-plane. J. Opt. Soc. Am. 73 (1983) 768-775
[5] Einstein FS, Juliano RA, DeAcetis LA, Lazar I: Experimental investigation of the far-field diffraction by a semiinfinite plane of variable thickness: Parallel polarization. J. Opt. Soc. Am. 69 (1979) 24-27

[6] Mata Mendez O, Cadilhac M, Petit R: Diffraction of a two-dimensional electromagnetic beam wave by a thick slit pierced in a perfectly conducting screen. J. Opt. Soc. Am. 73 (1983) 328-331

[7] Hamid MAK, Mohsen A, Boerner WM: Diffraction by a slit in a thick conducting screen. J. Appl. Phys. 40 (1969) 3882-3883

[8] Keller JB: Geometrical Theory of diffraction. J. Opt. Soc. Am. 52 (1962) 116-130

[9] Hönl H, Maue AW, Westpfahl K: Handbuch der Physik. XXV, 1. pp. 495-531. Springer-Verlag, Berlin 1961

[10] Born M, Wolf E: Principles of Optics. Pergamon Press, Oxford 1980 